\title{
How small is a small cloud?
}

\author{
I. Koren ${ }^{1}$, L. Oreopoulos ${ }^{2,3}$, G. Feingold ${ }^{4}$, L. A. Remer $^{3}$, and O. Altaratz ${ }^{1}$ \\ ${ }^{1}$ Department of Environmental Sciences Weizmann Institute, Rehovot 76100, Israel \\ ${ }^{2}$ Joint Center for Earth Systems Technology, University of Maryland, Baltimore County, Baltimore, Maryland, USA \\ ${ }^{3}$ Laboratory for Atmospheres, NASA Goddard Space Flight Center, Greenbelt, Maryland, USA \\ ${ }^{4}$ NOAA Earth System Research Laboratory, Boulder, Colorado, USA
}

Received: 1 February 2008 - Published in Atmos. Chem. Phys. Discuss.: 28 March 2008

Revised: 17 June 2008 - Accepted: 19 June 2008 - Published: 21 July 2008

Abstract. The interplay between clouds and aerosols and their contribution to the radiation budget is one of the largest uncertainties of climate change. Most work to date has separated cloudy and cloud-free areas in order to evaluate the individual radiative forcing of aerosols, clouds, and aerosol effects on clouds.

Here we examine the size distribution and the optical properties of small, sparse cumulus clouds and the associated optical properties of what is considered a cloud-free atmosphere within the cloud field. We show that any separation between clouds and cloud free atmosphere will incur errors in the calculated radiative forcing.

The nature of small cumulus cloud size distributions suggests that at any resolution, a significant fraction of the clouds are missed, and their optical properties are relegated to the apparent cloud-free optical properties. At the same time, the cloudy portion incorporates significant contribution from non-cloudy pixels.

We show that the largest contribution to the total cloud reflectance comes from the smallest clouds and that the spatial resolution changes the apparent energy flux of a broken cloudy scene. When changing the resolution from $30 \mathrm{~m}$ to $1 \mathrm{~km}$ (Landsat to MODIS) the average "cloudfree" reflectance at $1.65 \mu \mathrm{m}$ increases from 0.0095 to 0.0115 ( $>20 \%$ ), the cloud reflectance decreases from 0.13 to 0.066 $(\sim 50 \%)$, and the cloud coverage doubles, resulting in an important impact on climate forcing estimations. The apparent aerosol forcing is on the order of 0.5 to $1 \mathrm{Wm}^{-2}$ per cloud field.

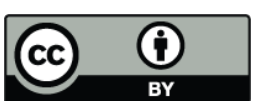

Correspondence to: I. Koren (ilan.koren@weizmann.ac.il)

\section{Introduction}

Clouds and aerosols have an important role in Earth's radiative energy budget and distribution. Both absorb and reflect incoming energy back to space. Clouds and aerosols interact and therefore influence one another's properties. The fraction of aerosols serving as cloud condensation nuclei (CCN) determines the cloud droplet size distribution, hence cloud optical properties, precipitation, cloud size, and lifetime. Likewise, cloud microphysical processes change aerosol size, distribution, chemical and optical properties. Light absorbing aerosols reduce the energy reaching the surface and warm the atmosphere. The warming aerosol layer stabilizes the atmosphere beneath it, reducing heat and moisture fluxes from the surface and consequently reduces shallow cloudiness (Koren et al., 2004; Feingold et al., 2005). Because a large fraction of aerosol is anthropogenic, it is important to estimate the net radiative forcing of man-made aerosols such as those from pollution and biomass burning, including aerosol indirect forcing through their influence on clouds.

To infer the atmospheric optical properties from spacebased observations, the atmosphere is classified as either cloudy or cloud-free. Cloud properties are retrieved from the cloudy pixels and aerosol properties are retrieved from the cloud-free pixels. It has been shown that the apparent "cloud-free" atmosphere within a cloud field, the "twilight zone", has unique optical properties pointing to contributions from undetected clouds and humidified aerosols (Koren et al., 2007; Charlson et al. 2007).

Moreover, it has been shown that the optical properties of the twilight zone are sensitive to the aerosol loading, suggesting the potential presence of anthropogenic forcing. Koren et al. (2007) examined this region using the ground-based AERONET remote sensing network in order to avoid the enhanced backscattering due to the presence of clouds (Wen et

Published by Copernicus Publications on behalf of the European Geosciences Union. 
Table 1. Information on the five Landsat scenes analyzed in this study.

\begin{tabular}{lrccccccccc}
\hline Name & Latitude & Longitude & date & SZA & CLF-LST & CLF-MOD & CLR-LST & CLR-MOD & BGR-LST & BGR-MOD \\
\hline The Bahamas & 24.56 & -74.61 & $10 / 20 / 1999$ & 41 & 0.1295 & 0.2612 & 0.1075 & 0.0489 & 0.0068 & 0.0096 \\
Barbados & 13.02 & -60.26 & $2 / 1 / 2002$ & 43 & 0.1015 & 0.2158 & 0.1422 & 0.0705 & 0.0161 & 0.0175 \\
Tuamotu Islands, & -15.91 & -145.24 & $7 / 26 / 2000$ & 48 & 0.1073 & 0.2438 & 0.0987 & 0.0425 & 0.0051 & 0.0063 \\
Polynesia & & & & & & & & & \\
Hawaii & 18.78 & -154.78 & $1 / 31 / 2001$ & 46 & 0.2279 & 0.3979 & 0.1372 & 0.0802 & 0.011 & 0.013 \\
100 km southeast of & -8.68 & -13.93 & $6 / 15 / 2001$ & 44 & 0.2409 & 0.4408 & 0.1632 & 0.0902 & 0.0084 & 0.0107 \\
Ascension Island & & & & & & & & & & \\
\hline
\end{tabular}

SZA - Solar Zenith angle

CLF-LST - Cloud fraction in the fine $(30 \mathrm{~m})$ resolution

CLF-MOD - Cloud fraction in the coarse $(960 \mathrm{~m})$ resolution

CLR-LST - Cloud average reflectance in the fine $(30 \mathrm{~m})$ resolution

CLR-MOD - Cloud average reflectance in the coarse $(960 \mathrm{~m})$ resolution

BGR-LST - Cloud-free average reflectance in the fine $(30 \mathrm{~m})$ resolution

BGR-MOD - Cloud-free average reflectance in the coarse $(960 \mathrm{~m})$ resolution

al., 2007). Charlson et al. (2007) did so using both airborne (downward-looking) lidar (20 m-200 m resolution) and the output from large eddy simulations of cloud fields with grid sizes of 50-70 m. Using independent methods, both groups identified the radiative importance of this inter-cloud region and the pitfalls of artificially separating "direct" (i.e., aerosol only) and "indirect" (i.e., cloud) radiative forcing.

Cloud size distributions for various cloud types studied previously (Plank, 1969; Wielicki and Welch, 1986; Rodts et al., 2003) have been found to obey power laws following analysis of observed marine stratocumulus, fair weather cumulus, and deep convective clouds (Cahalan and Joseph, 1989) and of modeled shallow cumulus clouds (Neggers et al., 2003; Xue and Feingold, 2006). For remotely-sensed clouds, it has been shown that the detector resolution may strongly affect the classification of cloudy and cloud-free pixels (Zhao and Di Girolamo, 2006) and that the contribution of small cumulus clouds to the total area is significant (Zhao and Di Girolamo, 2007). The resolution effect on the cloud-free area has been shown to decrease the likelihood of finding a cloud-free pixel from $16 \%$ to $3 \%$ when changing pixel size from $100 \mathrm{~km}^{2}$ to $10000 \mathrm{~km}^{2}$ (Krijger et al., 2007).

Here we study the contribution of a sparse field of small cumulus clouds to the radiation budget. We examine how small clouds contribute to the total reflectance as a function of their size distribution and how they affect the apparent cloud-free reflectance. The impact of sensor resolution on the optical property distribution of both the cloudy and cloud-free areas is examined as well. This has significant ramifications for the local estimates of aerosol direct and indirect forcing.

We define a sparse cumulus cloud field as one comprising many small shallow convective clouds distributed randomly (i.e., not clustered over a small portion of the field) with overall low cloud fraction $(<25 \%)$. Such a cloud field consists of many small $(10 \mathrm{~m}$ scale $)$ "stand-alone" clouds, namely clouds that form and develop into individual cells and are not derived from sheared patches of adjacent larger clouds. The condition of a small cloud fraction implies large average distances (compared to the cloud size) between detectable clouds. The requirement of high sparseness maximizes the interfacial area between clouds and the cloud-free atmosphere and therefore exhibits the strongest influence of the cloud twilight zone on the radiative forcing. Sparse cloud fields are common throughout the globe and can be found in marine trade cumulus systems, in the tropics and subtropics, and over rainforest areas subject to subsidence conditions where some of the humidity is supplied locally by evapotranspiration.

\section{Analysis}

To study the size distribution of sparse cloud fields we use channel $5(1.55-1.75 \mu \mathrm{m})$ data from the ETM+ radiometer on Landsat-7 (The source for this dataset was the Global Land Cover Facility, http://www.landcover.org) with a spatial resolution of $30 \mathrm{~m}$ (http://eros.usgs.gov/products/ satellite/landsat7.html). Channel 5 is used because the relatively long wavelength minimizes optical contributions of aerosols and atmospheric gases in cloud-free areas (Tanré et al., 1997), and the radiative effects of cloud-escapingphotons (the cloud 3-D effect, Wen et al., 2007).

Five cases of maritime, sparse cumulus cloud fields were selected away from sunglint to ensure the presence of low and uniform background reflectance and to facilitate the detailed cloud analysis (Table 1). The detectable cloud fraction ranges from $10 \%$ to $25 \%$. Clouds are identified using a reflectance threshold, which minimizes the contribution of the background far from clouds (Koren and Joseph, 2000). To achieve this, areas devoid of obvious detectable clouds at the margins of the cloud field were selected (with a minimum $5 \mathrm{~km}$ distance from detectable clouds). For these areas, the minimum reflectance threshold was selected to be three 

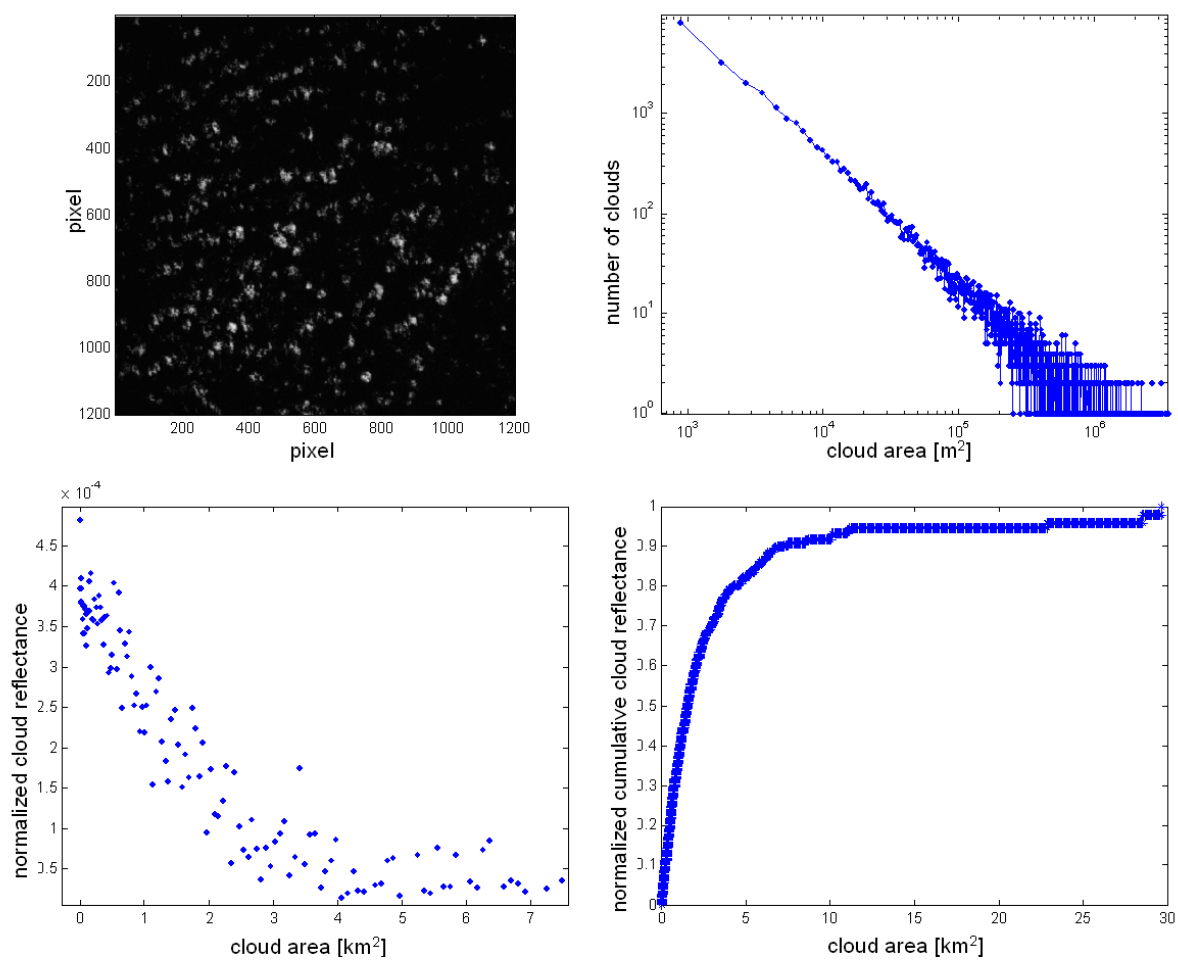

Fig. 1. Sparse cloud field over the Pacific Ocean near Tahiti. Top-left: Magnified portion of the cloud field with pixel resolution of $30 \mathrm{~m}$. Top-right: size distribution of the clouds. Note the large spread of the results for the large cloud sizes due to small number of samples in these bins. Bottom-left: normalized (unit integral) total per cloud area reflectance as a function of the cloud size. Bottom-right: cumulative cloud reflectance showing that most of the reflectance comes from the smaller clouds.

standard deviations above the mean, thus ensuring that more than $99.7 \%$ of the background is not selected. Since the solar IR channel used here is almost completely free of molecular and fine aerosol scattering, the calculated reflectance thresholds were consistent and low for all cases $(0.02 \pm 0.005)$. Pixels are considered to belong to the same cloud when two adjacent pixels are connected in any of the 8 possible directions.

Once the cloud mask is constructed for each cloud field, the cloud fraction, the average reflectance of the cloudy portion, and the cloud-free area, as well the average reflectance of the entire scene is calculated. Next, the area and average reflectance of each individual cloud is calculated and the distribution of cloud reflectance as a function of cloud size is inferred as the product of the average reflectance per unit cloud area, $r_{i}$, the cloud area, $a_{i}$ and the number of clouds per cloud size, $n_{i}$. Since the Sun-Satellite geometry is relatively constant over the area of the cloud field (typical lengths of $100 \mathrm{~km}$ ) the nadir reflectance observed by ETM+ is proportional to the radiative energy reflected back to space.

The total cloud reflectance $R$ (the sum over individual cloud sizes $i$ ) can be described as

$R=\sum_{i} R_{i}=\sum_{i} r_{i} n_{i} a_{i}$

To simulate the effect of resolution on the radiative distri- bution, the analysis is repeated five times with the resolution reduced successively by half, while ensuring that the total reflectance is conserved. Thus, a range of resolutions spanning the initial Landsat resolution of $30 \mathrm{~m}$ to a resolution close to that of MODIS $\left(30 \times 2^{5}=960 \mathrm{~m}\right)$ is achieved.

\section{Results}

In all five scenes the cloud size distribution exhibits a clear power-law behavior for clouds ranging from one pixel $\left(30 \times 30=900 \mathrm{~m}^{2}\right)$ to a few tens of $\mathrm{km}^{2}$ (Fig. 1), with an average correlation coefficient exceeding 0.95 (after logarithmic binning to correct for small number fluctuations in the large cloud bins; Newman, 2006). Such a distribution is described by a relationship with two scene-dependent parameters $b$ and $m$ :

$n(a)=\frac{b}{a^{m}}$,

where $n(a)$ is the number of clouds per given area $a$ (in pixels), $b$ is a constant equal to the number of clouds with an area of one pixel and $m$ is the power exponent of the distribution. The most notable properties of the power-law distribution are that the statistical moments of order $M$ are defined only for $M<m-1$ and that the distribution is scale invariant 

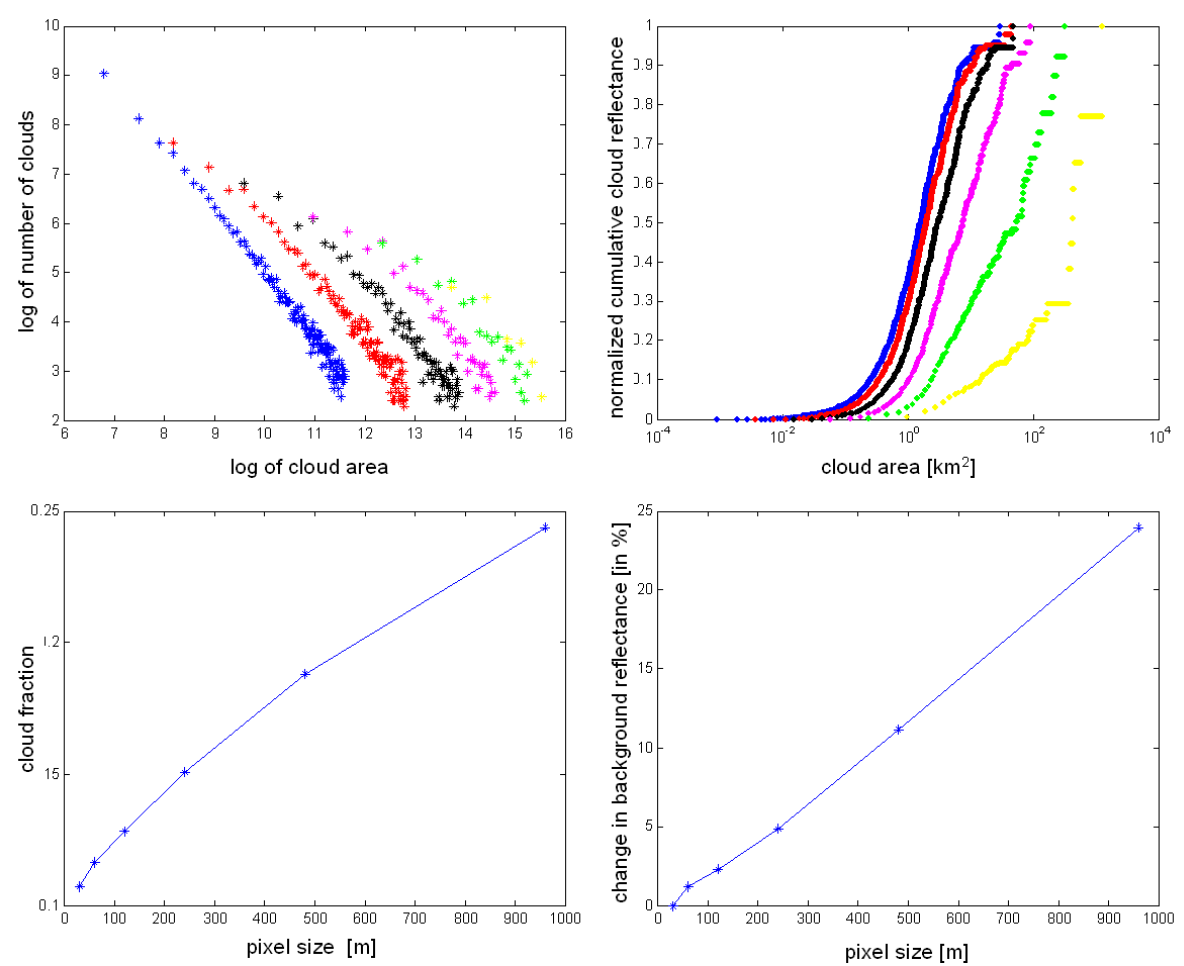

Fig. 2. The effect of resolution on the size distributions of a sparse cloud field over the Pacific Ocean near Tahiti. Top-left: log-log cloud size distributions for resolutions ranging from the original $30 \mathrm{~m}$ Landsat resolution (blue) to approximate $1 \mathrm{~km}$ MODIS resolution (yellow). Top-right: cumulative distribution of the cloud reflectance (normalized to unity) for all resolutions. Note how the size distribution (left) and the energy distribution (right) are shifted toward larger clouds as the resolution decreases. Bottom-left: change in cloud fraction from $12 \%$ in the highest to $24 \%$ in the coarsest resolution. Bottom-right: the effect of resolution on the "non-cloudy" pixels, showing a $24 \%$ increase in reflectance.

(Newman, 2006). The statistical moments are mean, variance, skewness etc for $M=1,2,3$ etc., respectively. The moment definition property implies that when $m<2$, the mean and all higher-order moments are ill-defined because they depend strongly on the limits of integration.

The total cloud area $A(a)$ covered by clouds with an area $a$ is given by:

$A(a)=a n=\frac{b}{a^{m-1}}$,

while the total reflectance $R(a)$ from clouds with an area $a$ and average reflectance $r$ can be calculated from:

$R(a)=r A$,

yielding a total cloud reflectance $R$ in the form of Eq. (1).

For all the analyzed cloud-size-distributions, the powerlaw exponent $m$ (the negative slope in log-log domain) ranges between 1 and $2(1.3 \pm 0.1)$. From this property of the distribution alone, one can gain three important insights into sparse cumulus cloud fields. First, a slope greater than 1 indicates that the area per cloud bin (Eq. 3) will also be powerlaw distributed with a negative slope $(0.3 \pm 0.1)$, and therefore that the total area of the small clouds will be larger than that of the large ones (Appendix A). Next, assuming that cloud area is a continuous variable, such a distribution suggests that small clouds below the detection limit of the Landsat instrument are more numerous than the detectable clouds. Lastly, a slope below 2 suggests that the mean area of clouds is not a well-defined distribution parameter (i.e., while it can be calculated for any given field, it is not a robust moment).

The reflectance distribution (Eq. 4) is a product of two functions with opposing trends. The total area per cloud size is larger for the small clouds ( $A(a)=b / a^{m-1}$ ) while the average reflectance is greater for larger (thicker) clouds. However, both the data and the analytical derivation (which can be found Appendix B) show that for our measured slopes of the power-law distribution, the total-area-function decreases faster than the total-reflectance-function increases with increasing cloud size. Therefore the reflectance contribution from the small clouds is greater than that of the larger ones (Fig. 1 lower panels). For all 5 scenes, most of the contribution to the total reflectance originates from the smaller clouds. Specifically, $15 \%$ to $50 \%$ of the reflectance (mean of $31 \%$ with standard deviation of $14 \%$ ) derives from clouds with areas below $1 \mathrm{~km}^{2}$. 

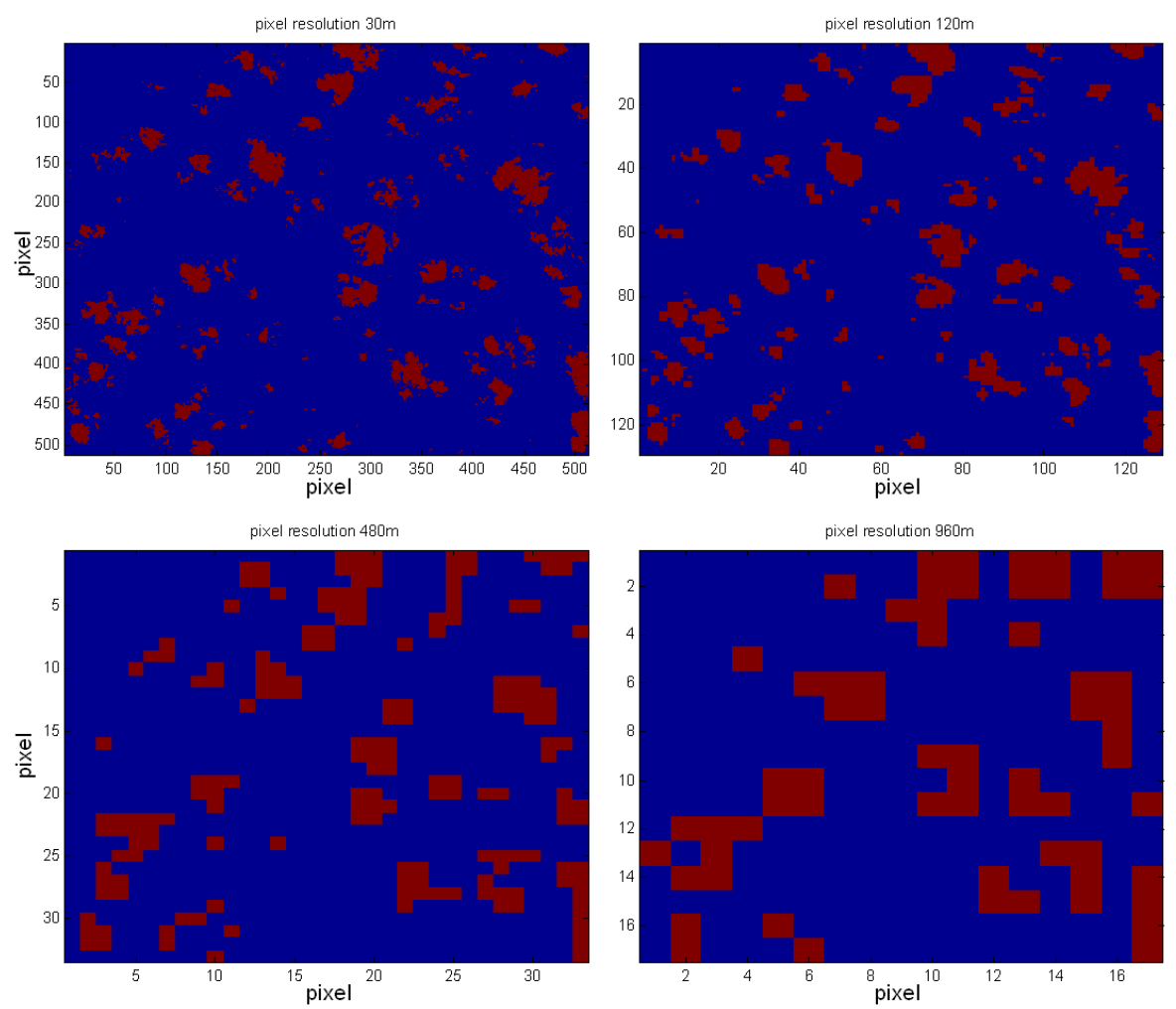

Fig. 3. Cloud mask for a sparse cumulus cloud field as inferred by using the same threshold at 4 different spatial resolutions. The upper-left panel is for the original Landsat resolution and the lower-right panel is for a MODIS-equivalent resolution. Note how the small clouds far from other clouds are "dissolved" into the background while small clouds, holes between clouds, and background pixels next to large cloud edges are all classified as clouds. The cloud fraction of the finer Landsat resolution is $11 \%$ and of the coarser MODIS resolution is $24 \%$. The mean cloud reflectance at the Landsat resolution is more than twice that at MODIS resolution which has $23 \%$ higher background reflectance.

The steep size distribution implies that the smaller clouds dominate the contributions to cloud number, total cloud area and reflected radiation. It is therefore expected that sensor resolutions much coarser than most clouds sizes of this type affect these properties of the cloud field as inferred from space. Indeed, upon reducing the resolution, the slopes of the distributions of all five fields become smaller (in absolute value) from an average of $1.3 \pm 0.1$ to $1.1 \pm 0.1$ showing shifting of the distribution toward the larger clouds (Fig. 2). These changes are due to averaging out the smaller clouds that are distant enough from other clouds and for which the average reflectance of the cloud and the surrounding background does not pass the threshold, while on the other hand, enlarging further the larger clouds by incorporating small holes and nearby clouds (Fig 3).

These changes in the size distribution of clouds drive changes in all other properties of the cloud field. The proportion of apparently cloudy to apparently cloud- free changes, as well as the optical properties of the clouds and background. The overall cloud fraction increases as large clouds become larger at a rate faster than that at which small clouds disappear into the apparent cloud-free zone. For all 5 fields the cloud fraction at MODIS resolution (mean 30\%, standard deviation, STD 11\%) is double that of the original Landsat resolution (mean 15\%, STD 6\%) (Table 1). The increased cloud fraction decreases the cloud-average reflectance to less than half, as more background pixels are classified as cloud. The background reflectance increases to an average of more than $23 \%$, as more isolated small clouds are "dissolved" into the "twilight zone" while the cloud reflectance distribution is shifted dramatically toward the larger clouds. Eventually, the reflectance appears to be mostly due to a few large clouds and assumes a completely different spatial distribution (Fig. 4).

How is the total energy redistributed in the coarse resolution? Even though the total reflectance is preserved when reducing the resolution, the pattern of radiative contributions from cloudy and cloud-free atmosphere changes dramatically. The end-result depends on the competition between the increase in total cloud area and the decrease in cloud-average reflectance with decreasing cloud size. Figure 4 (bottom) shows that for scenes with low initial cloud reflectance the total cloud reflectance decreases with decreasing resolution while for others it increases.

What is the apparent direct aerosol forcing due to the contribution of the small clouds misclassified as background? The aerosol forcing, defined as any deviation from the natural 

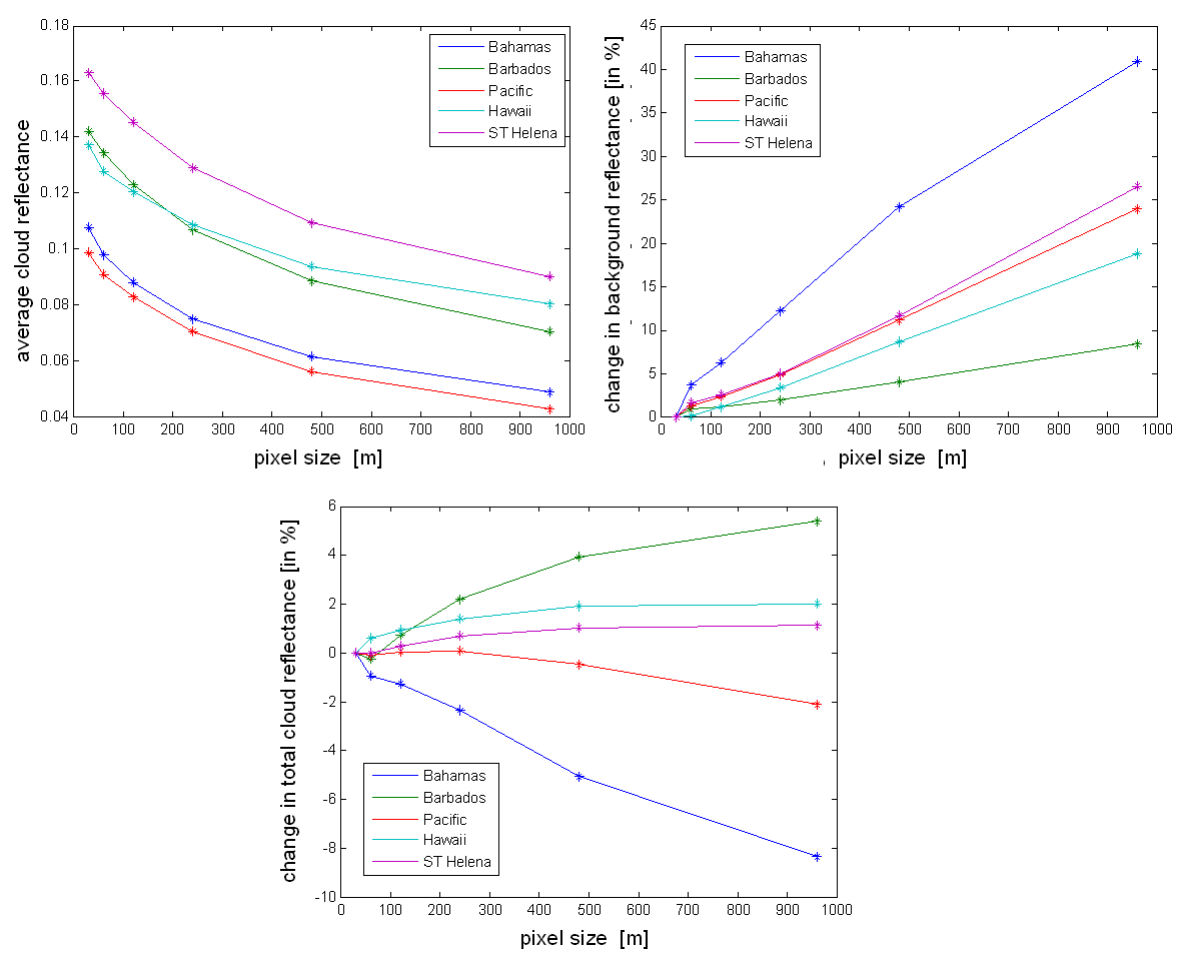

Fig. 4. The effect of resolution on all 5 sparse cloud fields. Top-left: average cloud reflectance. Top-right: changes in the background reflectance. Bottom: changes in total cloud energy (reflectance).

background, is calculated by comparing the real top-of-theatmosphere fluxes from the theoretical fluxes with (clean) background aerosol (Kaufman et al., 2001). Therefore for clean areas, the increase in background reflectance will be translated to a positive increase in the local aerosol loading. The mean change in the background reflectance between $30 \mathrm{~m}$ and $960 \mathrm{~m}$ is $0.0019 \pm 0.0006$. Since clouds have a relatively flat spectral dependence in solar wavelengths (shown for one case in Appendix C), this reflectance change corresponds to an apparent aerosol optical depth (AOD) of approximately 0.02 to 0.03 consisting of mostly coarse mode aerosol (Remer et al., 2005). This additional "artificial" AOD will be interpreted as an average $24 \mathrm{~h}$ forcing of $-0.8 \pm 0.2 \mathrm{~W} / \mathrm{m}^{2}$, or about $15 \%$ to $20 \%$ of the total global aerosol effect estimated from MODIS aerosol retrievals (Remer and Kaufman, 2006). Since small cumulus cloud fields are sparse, there are enough pixels labeled as cloud-free to meet the minimum amount of pixels per $10 \mathrm{~km}$ area to retrieve aerosols on most of the "cloud-free" area. We tested the 5 cases of this study and found that all of the MODIS AOD $10 \mathrm{~km}$ by $10 \mathrm{~km}$ pixel arrays had many more pixels than the minimum number, defined as cloud free.

While this may not seem to be a large forcing if it is only local, the large spatial coverage of marine sparse cumulus cloud fields (greater than $10 \%$ of all oceans and more than $16 \%$ of the area between $40 \mathrm{~N}$ to $40 \mathrm{~S}$ according Warren and Hahn, 2007) make it significant.

\section{Summary and conclusions}

We have shown that in a sparse cumulus cloud field, small clouds are extremely important. For all cases, the smallest detectable clouds contribute the most to the total cloud area as well as the mean field reflectance (Appendix B). The portion of small clouds that are "stand-alone", and far enough from other clouds, have the highest likelihood of being dissolved into the so-called twilight zone and to be labeled as "cloud-free" in coarser resolution imagery (Fig. 3).

We show that cloud sizes obey a power-law distribution with a slope bounded between 1 and $2(1<m<2)$. This finding, along with a reflection distribution and scale effect analysis imply that:

1. The distribution of the total area with respect to cloud size is also a power law with slope $(m-1) 0<m-1<1$ and therefore decreases monotonically as the cloud area increases (see Appendix A).

2. The reflectance per cloud size increases more slowly than the rate at which the cloud area per cloud size decreases. Therefore, small clouds contribute significantly to the total cloud reflectance (an analytical analysis and discussion are given in Appendix B). 


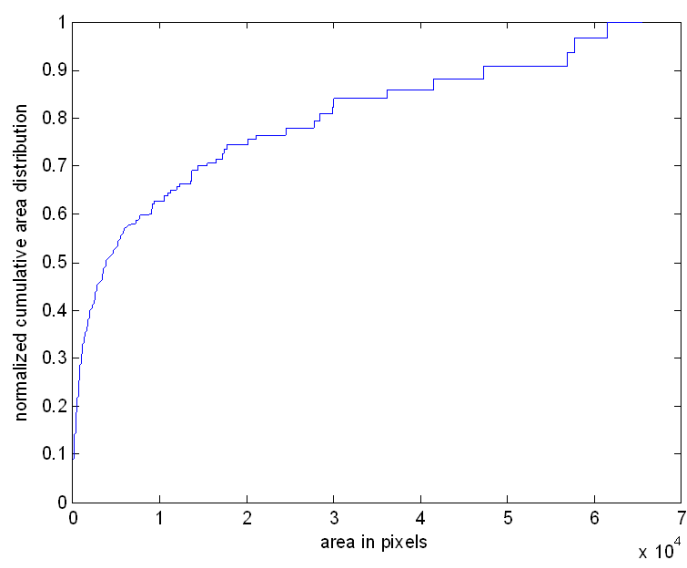

Fig. A1. Cumulative-distribution function of the total cloud area for the Ascension Island scene. $30 \%$ of the total cloud area comes from the first 1000 pixels (area of less than $1 \mathrm{~km}^{2}$ ).

3. The slopes of the distributions decrease with decreasing spatial resolution implying that an increasing amount of reflectance is falsely attributed to larger clouds.

4. The apparent direct aerosol forcing due to classifying cloud pixels as cloud-free in the sparse cumulus fields is on the order of $-0.8 \pm 0.2 \mathrm{Wm}^{-2}$ for the cloud field area. In reality the actual enhanced apparent forcing depends on the way clouds are detected in the algorithm. The specific MODIS errors in apparent direct aerosol forcing may be somewhat smaller than what is reported here due to the fact that clouds are detected by means of thresholds on the local variability in reflectance (Martins et al., 2002) rather than thresholds on the reflectance itself. Although we expect that the local variability of sub-pixel clouds will change similarly to the reflectance, cloud detection depends strongly on the thresholds (in reflectance or variability) and there may be cases where the variability method detects traces of sub-pixel clouds that the reflectance threshold will not.

5. For any resolution significant cloudy parts will be missed. This is an outcome of the nature of the powerlaw distribution of cloud areas. Since there is no physical limit at the highest Landsat resolution of $30 \mathrm{~m}$ this distribution may well extrapolate toward finer resolutions, suggesting that small clouds of only a few meters may contribute significantly to the total cloud fraction and reflectance.
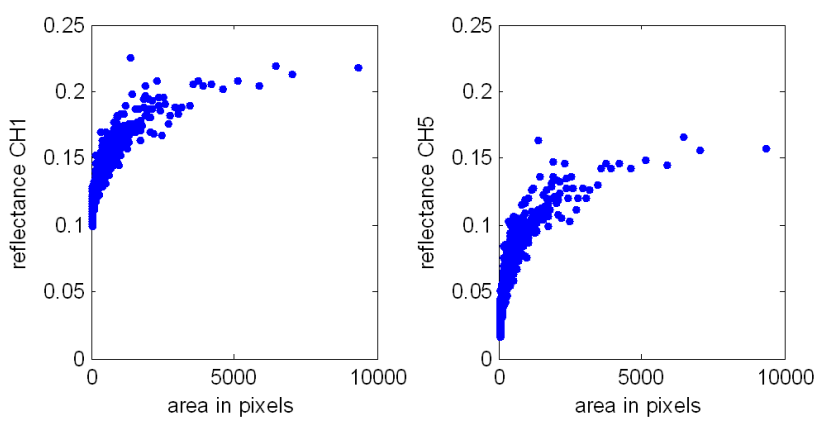

Fig. A2. Cloud reflectance as a function of the cloud area. The reflectance is defined here as the directional (nadir) radiance normalized by the downwelling solar irradiance in the particular band. The left panel is for the Landsat blue channel $(0.47 \mu \mathrm{m})$, and the right panel is for Landsat Band $5(1.63 \mu \mathrm{m})$.

\section{Appendix A}

\section{More on the power-law distribution of clouds}

In the paper we show that the size distribution of shallow marine cumulus clouds follows a power-law of the form:

$n(a)=\frac{b}{a^{m}}$,

where $n(a)$ is the number of clouds per given area $a$ (expressed in pixels for simplicity), $b$ is a constant equal to the number of clouds with an area of one pixel and $m$ is the power exponent of the distribution. From the above, the distribution of areas $A(a)$ covered by clouds with area $a$ is given by a power law, but with an exponent $m-1$ :

$A(a)=a n=\frac{b}{a^{m-1}}$,

In the paper we show that $m$ is bounded between 1 and 2 $(1<m<2)$. From these basic facts alone, one can infer essential information on the properties of the distribution of areas $A(a)$ and the moments of the distribution. Because the exponent of the area power-law is larger than zero $(0<m-1<1)$, the area $A(a)$ distribution will decay as clouds become larger which implies that small clouds contribute more to the total cloud area. Moreover, a cloud number distribution with $m>1$ (Eq. A1) implies that the number of clouds goes down rapidly (faster than 1/a) as their size increases. Figure A1 shows that $30 \%$ of the total cloud area comes from the first 1000 pixels (area of less than $1 \mathrm{~km}^{2}$ ) for the Ascension Island scene.

In the paper it is shown that the reflectance distribution is a product of two functions with opposing trends. The total area per cloud size is greater for the smaller clouds, while the average reflectance is greater for the larger (thicker) clouds (Fig. A2). 


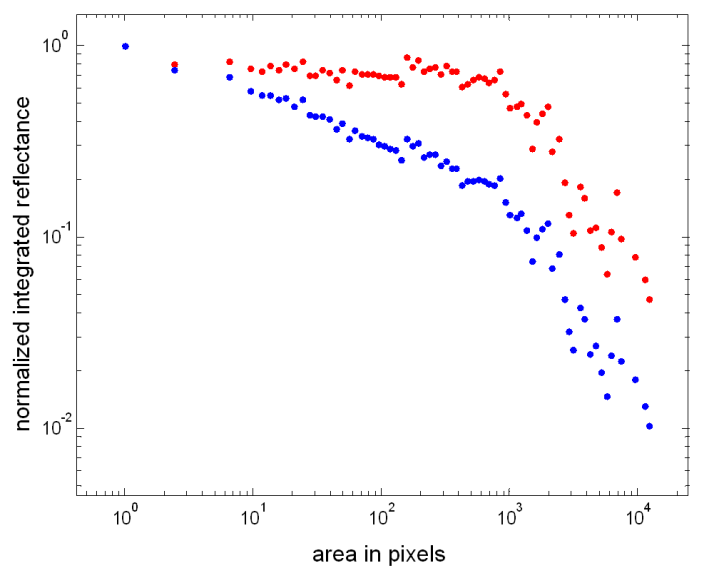

Fig. A3. Normalized total cloud reflectance as a function of cloud area for the Landsat blue channel $(0.47 \mu \mathrm{m}$, blue dots), and for Landsat Band $5(1.63 \mu \mathrm{m}$, red dots) after logarithmic binning to correct for small number fluctuations in the large cloud bins. Note that the reflectance of the smallest clouds is normalized to unity and in both cases contributes much more to the total cloud reflectance than the next available cloud size.

We define the unitless cloud-integrated reflectance $R$ as the reflectance from all clouds given by:

$$
R=\sum_{i} R_{i}=\sum_{i} r_{i} n_{i} a_{i}
$$

Summing reflectances of different clouds is permitted in our case because we deal with a relatively small area with uniform observational and solar geometry. The total reflectance so defined is thus used here as a direct measure of the total energy reflected back to space. When calculating the distribution of the total reflectance as a function of the cloud size, the contribution of the small clouds to the total reflectance is significant since the rate of decrease of the area is faster then the rate of increase of the reflectance as a function of cloud size. Figure A3 shows the reflectance distribution as a function of the cloud area. It is clearly shown that the "area trend" dominates the "reflectance trend". Therefore the reflectance of the smallest clouds contributes more to the total cloud reflectance.

\section{Appendix B}

\section{An analytical expression for the reflectance distribution function}

Assume that the optical thickness $\tau$ of a cloud is, amongst others, a function of its geometrical thickness $h$. If the cloud maintains an aspect ratio that is proportional to the cloud area we can write the following:

$\tau \propto h^{\beta}$

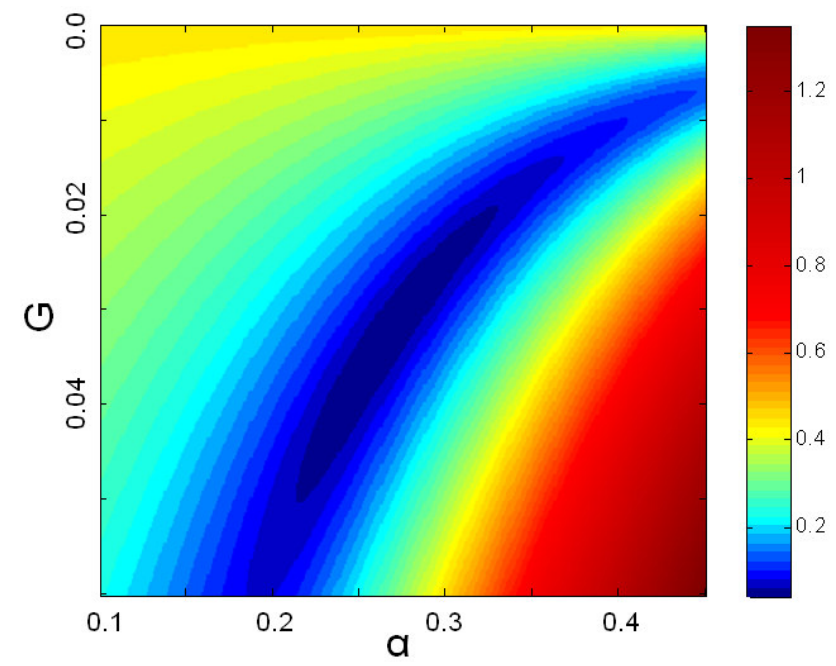

Fig. B1. Nadir reflectance error in $G-\alpha$ space for Band 5 of the Polynesian Landsat scene.

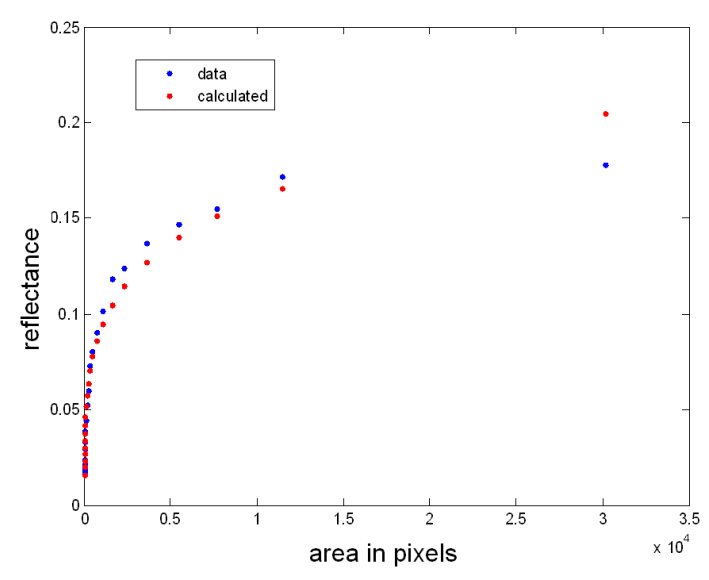

Fig. B2. Reflectance comparison for the optimal of $G$ and $\alpha$ value (those providing minimum reflectance error in $G-\alpha$ space).

$h \propto a^{1 / 2}$

For the general case of the aspect ratio not changing fast with cloud area, we can approximate the cloud optical thickness using the area with two free parameters $\left(c_{1}, \alpha\right)$ :

$\tau=c_{1} a^{\alpha}$,

where $\alpha=\beta / 2$. It can be shown that for the range of optical thicknesses between 0 and 20, appropriate for our shallow cumulus cloud fields, the nadir reflectance calculated with the multiple scattering code DISORT can be fit with a simple expression of the form:

$r=\frac{B \tau}{2+B \tau}$,

where $B$ assumes values approximately between 0.1 and 0.2 depending on the SZA and the exact values of asymmetry 
factor and single-scattering albedo of the cloud drop size distribution. This analytical expression is similar to the one for albedo in the two-stream approximation in the case of conservative scattering (Bohren, 1980).

Hence, the total reflectance per cloud area can be written as:

$R(a)=r n a=\frac{G a^{\alpha}}{2+G a^{\alpha}} b a^{(1-m)}$

where $G=B c_{1}$ is a collective parameter that includes all dependencies on solar zenith angle, cloud optical properties such as single-scattering albedo and asymmetry factor, and the proportionality parameter $c_{1}$ between the cloud area and $\tau$.

The conditions under which $R(a)$ becomes maximum can be explored by zeroing its first derivative with respect to cloud area:

$$
\begin{aligned}
\frac{d R}{d a} & =\frac{G b}{2+G a^{\alpha}}(1+\alpha-m) a^{(\alpha-m)} \\
& -\frac{G b}{\left(2+G a^{\alpha}\right)^{2}} a^{(1+\alpha-m)} G \alpha a^{(\alpha-1)} .
\end{aligned}
$$

For the derivative $\frac{d R}{d a}$ to become zero the following condition must be met:

$m-1=\frac{2 \alpha}{2+G a^{\alpha}}$.

The left hand side of the equation depends on the slope $m$ of the size (area) distribution and the right hand side depends on parameters related to the geometrical and optical properties of the clouds $(G$ and $\alpha$ ). The analytical reflectance function $R(a)$ reaches its maximum value when the area $a$ satisfies Eq. (B5). Note that the right hand side of Eq. B5 becomes maximum when the cloud area approaches zero and at this point depends only on $\alpha$, the exponent that translates area to $\tau$.

$m-1=\alpha$

Therefore, when $\alpha<m-1, R(a)$ does not have a maximum and decreases monotonically with increasing $a$, suggesting that the highest contribution to the reflectance comes from the smallest clouds (of 1 pixel).

Next, we use the data to estimate the empirical parameters $G$ and $\alpha$ by varying their values and comparing the theoretical reflectance calculations from Eq. (B2) to the actual (measured) reflectance as a function of the cloud area and seeking the values that minimize the error (in $L^{2}$ ). Figure B1 shows a clear, well-defined minimum suggesting that the agreement between theory and measurements occurs for an unambiguous point in $G-\alpha$ space. Figure B2 shows the reflectance comparison for the optimal $G$ and $\alpha$ values (those providing minimum reflectance error in $G-\alpha$ space).

For the Polynesian Landsat scene $G=0.0322$ and $\alpha=0.2690$ for band 5, and $G=0.173$ and $\alpha=0.125$ for band 1. Inserting the above values of $\alpha$ into Eq. (B6) shows that

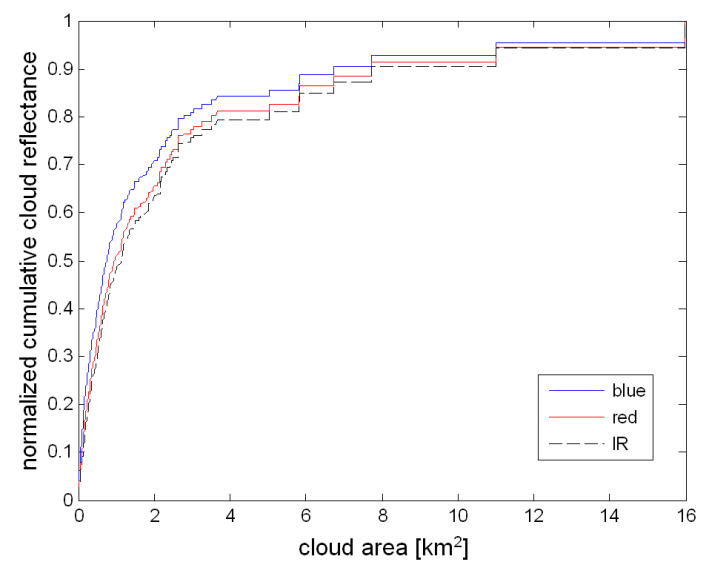

Fig. C1. Cumulative reflectance distribution as a function of cloud size for the Bahamas scene. Note that the reflectance contribution of the small clouds is sharper for the shorter wavelength.

any slope $m>1.269$ for band 5 and $m>1.125$ for band 1 will result in the clouds of smallest area contributing the most to the integrated reflectance. Indeed, this is clearly shown in the data (Fig. A3).

This method of retrieving $\alpha$ and $G$, statistically form the data can potentially be a powerful tool for structural and microphysical analysis of small clouds. In conjunction with the use of an absorbing and non-absorbing wavelength to retrieve the cloud optical thickness and the droplets effective radius on the pixel level (Nakajima and King, 1990), we retrieve $\alpha$ and $G$ statistically for the whole field, and link them to the cloud optical properties and geometry. While the retrieval of cloud optical thickness and droplets effective radius in isolated small clouds suffers from the 3-D effect (i.e. the breakdown of the plane parallel assumption for small clouds, Wen et al,. 2007) and small S/N ratio, $\alpha$ and $G$ are statistical parameters for the entire cloud field. These parameters can be retrieved for many wavelengths, absorbing and nonabsorbing, using the size distribution and any reflectance. Valuable information such as estimates of the cloud 3-D effects as a function of cloud size and aspect ratio, can be obtained from such an analysis (Koren et al., $2008^{1}$ ).

\section{Appendix C}

\section{Forcing analysis for the Landsat's blue and red wavelengths}

We performed the analysis in the NIR $(1600 \mathrm{~nm})$ to $\mathrm{min}$ imize the contribution of molecular scattering, aerosols and cloud 3-D effects (Wen et al., 2007). Also, the Landsat NIR channel (ch-5) is known to be superior

\footnotetext{
${ }^{1}$ Koren, I., et al.: A new approach to statistical inversion of microphysical properties of scattered cumulus cloud field, in preparations for Atmos. Chem. Phys., 2008.
} 
Table C1. The resolution effect in the blue, red and NIR Landsat channels.

\begin{tabular}{ccc}
\hline $\begin{array}{c}\text { Wavelength } \\
\mathrm{nm}\end{array}$ & $\begin{array}{c}\text { Background reflectance } \\
\text { difference* }^{*}\end{array}$ & $\begin{array}{c}\text { Mean cloud reflectance } \\
\text { difference }^{* *}\end{array}$ \\
\hline 480 & 0.0026 & 0.0532 \\
660 & 0.0028 & 0.0597 \\
1600 & 0.0028 & 0.0587 \\
\hline
\end{tabular}

* Differences in background reflectance between $960 \mathrm{~m}$ and $30 \mathrm{~m}$ resolution.

** Differences in the mean cloud reflectance between $30 \mathrm{~m}$ to $960 \mathrm{~m}$ resolution.

with respect to signal-to-noise (http://imaging.geocomm. com/features/sensor/landsat7). We nevertheless repeated the analysis for the Landsat blue $(480 \mathrm{~nm})$ and red $(660 \mathrm{~nm})$ channels of the Bahamas scene. The results are shown in Table C1.

As discussed above, the reflectance distribution combines the contributions of two functions with opposing tendencies. The total cloud area per cloud size goes down fast (as a power-law function) for the large clouds while the reflectance goes up. Figure $\mathrm{C} 1$ shows the cumulative reflectance as a function of the cloud size for all 3 wavelengths.

Acknowledgements. This paper is dedicated to the memory of Yoram J. Kaufman, a dear friend and a brilliant scientist. This research was supported in part by the Israel Science Foundation (grant 1355/06) and NASA's Radiation Sciences Program and Interdisciplinary Studies. We thank the reviewers for helpful comments. I. $\mathrm{K}$. is incumbent of the Benjamin H. Swig and Jack D. Weiler career development chair.

The source for this dataset was the Global Land Cover Facility, http://www.landcover.org

\section{References}

Cahalan, R. F. and Joseph, J. H.: Fractal Statistics of Cloud Fields, Mon. Weather Rev., 117, 261-272, 1989.

Charlson, R. J., Ackerman, A. S., Bender, F. A.-M., Anderson, T. L., and Liu, Z.: On the climate forcing consequences of the albedo continuum between cloudy and clear air, Tellus, 59B, 715-727, doi:10.1111/j.1600-0889.2007.00297.x., 2007.

Feingold, G., Jiang, H., and Harrington, J. Y.: On smoke suppression of clouds in Amazonia, Geophys. Res. Lett., 32, 2, L02804, doi:10.1029/2004GL021369, 2005.

Kaufman, Y. J., Smirnov, A., Holben, B., and Dubovik, O.: Baseline maritime aerosol methodology to derive the optical thickness and scattering propertiesm, Geophys. Res. Lett., 28, 3251-3254, 2001.

Koren, I. and Joseph, J. H.: The histogram of the brightness distribution of clouds in high resolution remotely sensed images, J. Geophys. Res., 105(D24), 29369-29377, 2000.

Koren, I., Kaufman, Y. J., Remer, L. A., and Martins, J. V.: Measurement of the Effect of Amazon Smoke on Inhibition of Cloud Formation, Science, 303, 1342-1345, 2004.
Koren, I., Remer, L. A., Kaufman, Y. J., Rudich, Y., and Martins, J. V.: On the twilight zone between clouds and aerosols, Geophys. Res. Lett., 34, L08805, doi:10.1029/2007GL029253, 2007.

Krijger, J. M., van Weele, M., Aben, I., and Frey, R.: Technical Note: The effect of sensor resolution on the number of cloud-free observations from space, Atmos. Chem. Phys., 7, 2881-2891, 2007 , http://www.atmos-chem-phys.net/7/2881/2007/.

Martins, J. V., Tanre, D., Remer, L. A., Kaufman, Y. J., Mattoo, S., and Levy, R.: MODIS cloud screening for remote sensing of aerosol over oceans using spatial variability, Geophys. Res. Lett., 29, 8009, doi:10.1029/2001GL01352, 2002.

Nakajima, T. and King, M. D.: Determination of the optical thickness and effective particle radius of clouds from reflected solar radiation measurements. Part I: Theory, J. Atmos. Sci., 47, 1878 1893, 1990.

Neggers, R. A. J., Jonker, H. J. J., and Siebesma, A. P.: Size statistics of cumulus cloud populations in large-eddy simulations, J. Atmos. Sci., 60, 1060-1074, 2003.

Newman, M. E. J.: Power laws, Pareto distributions and Zipf's law, Contemp. Phys., 46, 5, 323-351, 2005.

Plank, V. G.: The Size Distribution of Cumulus Clouds in Representative Florida Populations, J. Appl. Meteor., 8, 46-67, 1969.

Remer, L. A., Kaufman, Y. J., Tanre, D., Mattoo, S., Chu, D. A., Martins, J. V., Li, R. R., Ichoku, C., Levy, R. C., Kleidman, R. G., Eck, T. F., Vermote, E., and Holben, B. N.: The MODIS aerosol algorithm, products and validation, J. Atmos. Sci., 62, 947-973, 2005.

Remer, L. A. and Kaufman, Y. J.: Aerosol direct radiative effect at the top of the atmosphere over cloud free ocean derived from four years of MODIS data, Atmos. Chem. Phys., 6, 237-253, 2006, http://www.atmos-chem-phys.net/6/237/2006/.

Rodts, S. M. A., Duynkerke, P. G., and Jonker, H. J. J.: Size distributions and dynamical properties of shallow cumulus clouds from aircraft observations and satellite data, J. Atmos. Sci., 60(16), 1895-1912, 2003.

Warren, S. G. and Hahn, C. J.: Climatic Atlas of Clouds Over Land and Ocean, http://www.atmos.washington.edu/CloudMap/ (last access: 21 July 2008), 2007.

Tanre, D., Kaufman, Y. J., Herman, M., and Mattoo, S.: Remote sensing of aerosol properties over oceans using the MODIS/EOS spectral radiances, J. Geophys. Res.-Atmos., 102, 1697116988, 1997.

Wielicki, B. A. and Welch, R. M.: Cumulus Cloud Properties Derived Using Landsat Satellite Data, J. Appl. Meteor., 25, 261$276,1986$.

Wen, G., Marshak, A., Cahalan, R. F., Remer, L. A., and Kleidman, R. G.: 3D aerosol-cloud radiative interaction observed in collocated MODIS and ASTER images of cumulus cloud fields, J. Geophys. Res., 112, D13204, doi:10.1029/2006JD008267, 2007.

Xue, H. and Feingold, G.: Large-Eddy Simulations of Trade Wind Cumuli: Investigation of Aerosol Indirect Effects, J. Atmos. Sci., 63(6), 1605-1622, doi:10.1175/JAS3706.1, 2006.

Zhao, G. and Di Girolamo, L.: Cloud fraction errors for trade wind cumuli from EOS-Terra instruments, Geophys. Res. Lett., 33, L20802, doi:10.1029/2006GL027088, 2006.

Zhao, G. and Di Girolamo, L.: Statistics on the macrophysical properties of trade wind cumuli over the tropical western Atlantic, J. Geophys. Res., 112, D10204, doi:10.1029/2006JD007371, 2007. 